\title{
Correction to: Relationship between periodontal parameters and plasma cytokine profiles in pregnant woman with preterm birth or low birth weight
}

\author{
Francisco Mesa ${ }^{1} \cdot$ Elena Pozo $^{1} \cdot$ Francisco O’ Valle $^{2} \cdot$ Alberto Puertas $^{3} \cdot$ Antonio Magan-Fernandez $^{1} \cdot$ Eva Rosel $^{4} \cdot$ \\ Manuel Bravo 4
}

Published online: 3 September 2020

(C) Springer-Verlag GmbH Germany, part of Springer Nature 2020

\section{Correction to: Clinical Oral Investigations https://doi.org/10.1007/s00784-015-1553-x}

The authors are correcting this article because they accidentally included some wrong data in the analysis.

The first paragraph of Results should now be worded as follows:

“... In the control group, the mean age was $29.5 \pm 5.0$ years, mean gestation period $39.7 \pm 1.1$ weeks, and the mean newborn weight was $3371 \pm 417 \mathrm{~g}$. In the case group, the mean age was $29.6 \pm 6.1$ years, mean gestation $35.4 \pm 1.8$ weeks, and mean newborn weight $2343 \pm 414 \mathrm{~g}$; in this group, 17 children had normal weight $(\geq 2500 \mathrm{~g}$ ) but $<37$ weeks of gestation, 8 children weighed $<2500 \mathrm{~g}$ but were full-term deliveries, while 42 children weighed $<2500 \mathrm{~g}$ and were preterm deliveries".

The second paragraph of results should be deleted ("Although the difference in CA loss between cases and 31 controls was significant in the unadjusted analysis, statistical 32 significance was lost after adjustment for socioeconomic level 33 and tobacco use $(p=0.249) . ")$

The modified Table 1 (below) should exchange Table 1.

The online version of the original article can be found at https://doi.org/ 10.1007/s00784-015-1553-x

Francisco Mesa

fmesa@ugr.es

1 Periodontology Department, School of Dentistry, University of Granada, Campus de Cartuja s/n, 18071 Granada, Spain

2 Pathology Department, School of Medicine, University of Granada, Avda. De Madrid s/n, 18012 Granada, Spain

3 Department of Obstetrics and Gynecology, Virgen de las Nieves University Hospital, Avda. Fuerzas Armadas 2, 18014 Granada, Spain

4 Department of Preventive Dentistry and Epidemiology, School of Dentistry, University of Granada, Campus de Cartuja s/n, 18071 Granada, Spain 
Table 1 Description and comparison of the socio-demographic and periodontal variables of the pregnant women $(n=131)$

\begin{tabular}{|c|c|c|c|}
\hline Variable & Controls $(n=64)$ & Cases $(\mathrm{PB} / \mathrm{LBW})(n=67)$ & $P$-values \\
\hline Ethnicity (\% Caucasian) & 82.8 & 83.6 & $0.908^{\mathrm{a}}$ \\
\hline Marital status (\% married) & 73.4 & 58.2 & $0.098^{\mathrm{a}}$ \\
\hline Employed (\% yes) & 45.3 & 40.3 & $0.687^{\mathrm{a}}$ \\
\hline Social level (range, I-VII), median & VII & VII & $0.484^{\mathrm{b}}$ \\
\hline Schooling* (years) & $11.5 \pm 4.9$ & $10.1 \pm 4.2$ & $0.074^{\mathrm{c}}$ \\
\hline Coffee ( $\%$ No-one cup/week-1 cup daily) & $87-13-0$ & $69-31-0$ & $0.017^{\mathrm{a}}$ \\
\hline Tobacco $(\%$ never/ex-smoker- $\leq 10 /$ day- $>10 /$ day $)$ & $77-3-6-14$ & $55-22-6-16$ & $0.039^{\mathrm{b}}$ \\
\hline Alcohol ( $\%$ None- $\leq 5 \mathrm{~g} /$ day- $>5 \mathrm{~g} /$ day $)$ & $100-0-0$ & $99-1-0$ & $\approx 1^{\mathrm{d}}$ \\
\hline Gestational diabetes $(\%)$ & 3.1 & 6.0 & $0.681^{\mathrm{d}}$ \\
\hline Arterial hypertension (\%) & 7.8 & 3.0 & $0.267^{\mathrm{d}}$ \\
\hline Genitourinary infections (\%) & 20.3 & 31.3 & $0.215^{\mathrm{a}}$ \\
\hline Chorioamnionitis (\%) & 0.0 & 1.5 & $\approx 1^{\mathrm{d}}$ \\
\hline Premature membrane rupture $(\%)$ & 7.8 & 50.7 & $<0.001^{\mathrm{a}}$ \\
\hline Gingival bleeding index* $(\%)$ & $19.3 \pm 26.7$ & $23.7 \pm 31.1$ & $0.608^{\mathrm{c}}$ \\
\hline Gingival recession* $(\mathrm{mm})$ & $0.05 \pm 0.12$ & $0.10 \pm 0.24$ & $0.173^{\mathrm{c}}$ \\
\hline Probing depth* $(\mathrm{mm})$ & $1.66 \pm 0.46$ & $1.76 \pm 0.62$ & $0.272^{\mathrm{c}}$ \\
\hline Clinical attachment loss* (mm) & $1.71 \pm 0.53$ & $1.86 \pm 0.82$ & $0.206^{\mathrm{c}}$ \\
\hline Patients with Periodontitis (\%) & 6.3 & 16.4 & $0.121^{\mathrm{a}}$ \\
\hline
\end{tabular}

a Chi-square test with Yates' correction.

b Mann-Whitney $U$ test.

c Student's $t$ test,

d'Bilateral Fisher's exact test

*Values are expressed as mean \pm standard deviation.

Publisher's note Springer Nature remains neutral with regard to jurisdictional claims in published maps and institutional affiliations. 